\title{
Response bias and hemispheric differences in dot localization
}

\author{
M. P. BRYDEN \\ University of Waterloo, Waterloo, Ontario, Canada N2L \$G1
}

\begin{abstract}
A series of studies investigated the accuracy of dot localization in left and right visual fields. The left visual field superiority previously reported for this task was found to be small and statistieally inconsistent. It was unaffected by the presence of a frame of reference, the location of the response card, or the sex of the subject. It was observed, however, that the majority of subjects showed a response bias favoring the report of items in the left visual field. This suggests that the right hemisphere is less critical than the left in its assessment of incoming information, and may provide a basis for understanding previous reports of right hemispheric superiority on dot localization. It is concluded that dot localization does not provide a reliable measure of right hemisphere performance.
\end{abstract}

Experiments on normal adults have revealed both a right visual field superiority for verbal material and a left visual field superiority on a wide variety of spatial and other nonverbal tasks (Heron, 1957; White, 1969). By and large, the verbal right-field effects have been fairly robust. while the nonverbal left-field effects have been smaller and less readily replicable (Kinsbourne, 1973; White, 1972). Yet the development of an adequate test of right hemispheric function in normal individuals is a necessary precursor to the study of interhemispheric relationships and of the ontogeny of cerebral asymmetry. Milner (1974), for example, has espoused a principle of "complementary specialization": for the normal individual, this implies that left hemispheric specialization for verbal processes goes hand in hand with a right hemispheric specialization for spatial or nonverbal processes. Without a reliable measure of nonverbal functioning, this concept cannot be put to the test.

The present report deals with one task that has frequently been cited as providing an appropriate measure of right hemispheric performance, that of localizing a point in two-dimensional space. Kimura (1969) reported a series of studies in which a left visual field superiority for dot localization was found. When the dot was enclosed by one of two squares appearing to the left or right of tixation, men showed a left visual field superiority but women did not. When the field was enclosed by a single circle centered at fixation, both sexes showed the same left visual field effect. In a subsequent study, Pohl, Butters, and Goodglass (1972) were unable to replicate Kimura's findings, but found a right visual field superiority when no

This research was supported by Grant A-95 from the National Research Council of Canada. Thanks are due to Anne Henderson and Diane Couture for their assistance in data collection and analysis. and to Fran Allard for her critical comments. surrounding frame was employed. Bryden (1973) also used no surrounding frame, but found no reliable visual field differences at all.

Thus, the dot localization task provides a plethora of results, like many other visual procedures that have been purported to provide an assessment of right hemispheric function in normal subjects (see White, 1972, Table 1, p. 499). The present series of experiments represents an attempt to understand the factors underlying performance on dot localization tasks.

\section{EXPERIMENT I}

The procedures that have been employed in previous dot localization studies have varied widely. Both Kimura (1969) and Pohl et al. (1972) presented a frame around the area in which the dot might appear, while Bryden (1973) did not. Furthermore, Bryden's subjects were presented the response matrix in the same physical location as the dot had appeared, while in the other studies, subjects had to move their heads to look at a response card outside the tachistoscope.

Looking at a response card outside the tachistoscope requires a head movement, therefore a change of reference point and a constancy scaling operation. since the response card is at a different distance from the subject than the stimulus card. Therefore, two conditions were employed in this first experiment: one in which the subject looked outside the machine to a response card and one in which the response card appeared in the tachistoscope after a 1 -sec delay. Since the visual field of the tachistoscope employed was quite circumscribed and provided a natural frame around the area in which dots might appear, no physical frame was employed. Thus, the general procedure was most closely analogous to that employed by Pohl et al. (1972) in their first 


\begin{tabular}{|c|c|c|c|c|c|c|c|c|c|c|c|c|c|}
\hline 74 & 26 & 10 & $\mathrm{tt}$ & 53 & 48 & 69 & 14 & 35 & 97 & $\mathrm{nn}$ & 02 & 81 & 49 \\
\hline 88 & 39 & 47 & 62 & 00 & 16 & $\mathrm{ff}$ & 90 & 57 & 31 & 24 & 19 & 65 & $x x$ \\
\hline $2 z$ & 05 & 198 & 28 & 16 & 79 & 34 & 52 & 83 & 68 & 16 & 80 & 73 & 08 \\
\hline 82 & 13 & 39 & 104 & 18 & 55 & 86 & $\mathrm{rr}$ & 60 & 9 & 88 & 11 & 94 & 45 \\
\hline 95 & 22 & 84 & 2 & 15 & 30 & 77 & 93 & 42 & os & 46 & 67 & 56 & 70 \\
\hline $\mathrm{hh}$ & 61 & 30 & 6 & s. & 27 & 01 & $\mathrm{dd}$ & 17 & 75 & 80 & 8 & 98 & bb \\
\hline 76 & 29 & 44 & 06 & 23 & 50 & $\mathrm{cc}$ & 71 & 33 & 46 & 21 & 59 & 07 & 85 \\
\hline 40 & 12 & ss & 78 & 63 & 87 & 25 & 09 & 68 & 36 & 80 & kk & 54 & 96 \\
\hline
\end{tabular}

Figure 1. The response card. In Experiments I and II, test dots appeared only in the shaded areas. In addition, in Experiments III-IV, response numbers appeared only in the shaded areas.

experiment. in which a right visual field superiority was obtained, and by Bryden (1973), who observed no laterality effect.

\section{Method}

Materials. The visible field of the tachistoscope employed was approximately $7 \times 4$ in. A response card was constructed by dividing this area into $112 \frac{1}{2}$-in. squares, in a $14 \times 8$ matrix. These squares were then numbered in random sequence with two-digit numbers or with lowercase letter pairs, as shown in Figure 1. When viewed in the tachistoscope, each square subtended a visual angle of approximately $0.59^{\circ}$ in both vertical and horizontal dimensions.

Stimulus cards were prepared so that a small black dot would appear at a position corresponding to the center of one of the squares of the response card. In actual fact, test dots appeared in only 24 of the 112 possible positions. The positions used were those at least two squares away from the edge of the card and at least two squares away from the fixation point. This provided a total of 12 possible positions in each visual field. These positions are indicated by the shaded areas in Figure 1. Dots ranged in distance from about $1.5^{\circ}$ from fixation to about $2.8^{\circ}$. In addition, 12 other dot cards, with the dots randomly positioned outside the shaded areas. were employed in the experiment, but responses to them were not scored.

Subjects. Subjects for this experiment were 40 introductory psychology students, 20 men and 20 women, at the University of Waterloo. All were right-handed. Twenty subjects, 10 men and 10 women, were assigned to each of the two groups.

Procedure. The material was presented in a Scientific Prototype three-channel tachistoscope (Model GB), at an illumination of approximately $23 \mathrm{fL}$. The subjects were instructed to look at a central fixation point when a verbal "ready" signal was given. A single dot was then exposed for $10 \mathrm{msec}$. Subjects in Group 1 then removed their heads from the tachistoscope visor and looked at a response card such as that shown in Figure 1, which was situated in a holder on the top of the tachistoscope box. The subjects were required to indicate the number of the square in which the dot had appeared. A response was required on every trial.

Subjects in Group 2 were given the same general instructions, except the response card appeared in the tachistoscope field $1 \mathrm{sec}$ after the dot had been shown and was visible for a timed 5-sec interval. During this delay interval, the fixation point was in view and the field was illuminated.

All subjects were given a total of 60 trials. On 24 trials a dot appeared within the circumscribed test area of the left visual field, on 24 trials a dot appeared in the test area of the right visual field. and on 12 trials a dot appeared in other positions, randomly selected, outside the test area. These latter 12 trials were not scored. Thus, every subject was scored on 48 trials.
In each block of 10 trials. four test dots appeared in the left visual field and four in the right visual field; and there were two buffer trials.

Scoring. The subjects were scored as correct for each test dot they localized in the correct square. In a secondary analysis, an error score based on the distance between the square called by the subject ind the square in which the dot actually appeared was employed. This measure yielded fundamentally the same information as that obtained with the absolute correct measure. However, the error measure frequently showed large deviations resulting from apparently random guesses. As a consequence, only the absolute correct measure will be reported in this paper.

\section{Results}

An overall analysis of variance showed no significant laterality effect $[F(1.36)=1.67, p>.20]$. The only significant effect was a difference between the two conditions in overall accuracy $[F(1,36)=$ 8.34. $\mathrm{p}>.01$ ]: The subjects were much more accurate when the response card appeared in the tachistoscope than when they had to look outside the machine. Sex differences were not significant, though there was a tendency for men to be more accurate when the response card was in the machine and the women to be more accurate when it was outside $[F(1,36)=$ 2.72. $p<.20$ ]. In general, there was a slight trend otwards a left visual field superiority, as shown in Table 1.

If one looks instead at the proportion of subjects showing a particular visual field superiority (Table 1). one again finds no significant laterality effects. This corroborates the results of the analysis of variance.

The results of this experiment can hardly be described as overwhelming support for previous findings in the area. It is Group 1, where subjects had to look away from the tachistoscope to see the response card. that most closely resembles the procedure used by Pohl et al. (1972). They found a right visual tield superiority, while the present study found a slight left-field superiority. Certainly the effect observed here is not as great as that reported by Kinura (1969). who used a frame around the stimulus field. On the basis of the overall analysis of variance. it is perhaps safest to conclude that there is no very convincing evidence for any laterality effect with the procedures employed.

\section{Table 1}

Correct Localizations in Left and Right Visual Fields: Experiment I

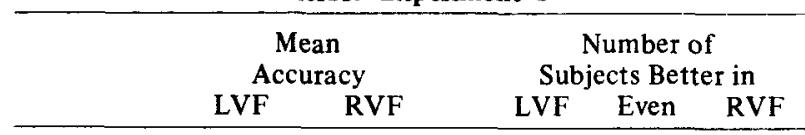

\section{Group 1*}

Women

Total

4.2

4.2
7.1

5.7

4.4

5.0

Group 2**

Men

Women

11.3

Total

8.5

9.9

4.7

$\begin{array}{rll}4 & 3 & 3 \\ 6 & 0 & 4 \\ 10 & 3 & 7\end{array}$

*Response card outside

\begin{tabular}{rrrr}
10.1 & 6 & 1 & 3 \\
7.1 & 6 & 2 & 2 \\
8.6 & 12 & 3 & 5 \\
\hline
\end{tabular}

**Response card in T-scope 
Of course. it is difficult to interpret results when one has not observed a statistically reliable effect. However. the present study employed virtually the same number of trials in each visual field as Kimura's (1969) study. Absolute accuracy was intentionally kept low in the hopes of utilizing the deviation error score. but essentially the same results were obtained with both measures. Finally. Blumstein. Goodglass, and Tartter (1975) indicate that about $80 \%$ of the right-handed subjects show a right-ear effect in dichotic listening experiments: one would expect a useful index of visual laterality to show the same consistencr.

\section{EXPERIMENT II}

In the preceding experiment, dots were presented in an otherwise blank field. Pohl et al. (1972) have made much of whether or not a frame of reference is present in the visual field. considering the task one of personal spatial'discrimination when a blank field is used and one of extrapersonal spatial discrimination when a frame of reference appears. They report a right visual field superiority with a blank field and no laterality effect with a frame of reference. The original Kinura (1969) study, however. used a frame of reference and obtained a left visual field effect. In order to pursue the differences between these two conditions. the second experiment involved a replication of the first. but with a frame added around the area in which the test dots appeared.

\section{Method}

Materials. Stimulus cards were the same as those used in the preceding experiment. except that the 12 cards with dots outside the test area were discarded. The fixation card was changed so that it included two outline rectangles enclosing the areas to the left and right of fixation in which the dots would appear. Likewise. the response card was moditied to provide responses for only the 24 positions actually used.

Subjects. The subjects were 40 right-handed volunteers drawn from the introductory psychology classes at the Liniversity of Waterloo. Twenty subjects. 10 men and 10 women. were assigned to each of two groups. As in Experiment 1. one group was tested with the response card outside the tachistoscope and one with it in the machine.

Procedure. Presentation conditions were identical to those emplosed in the first experiment. Since there were no buffer trials. each subject received only 48 trials. 24 with a dot in the left visual field and 24 with a dot in the right visual field. Within each block of 8 trials, four dots appeared to the lefi of fixation and four to the right.

\section{Results}

The results of this experiment are shown in Table 2. An analysis of variance indicated no significant effects. although there was a slight trend for accuracy to be greater when the response card was in the machine $[F(1.36)=2.84, p=.10]$. None of the other effects even approached the .10 level of confidence. As in Experiment I. a measure of deviation error vielded essentially similar results.
Table 2

Correct Localizations in Left and Right Visual Fields in the Presence of a Frame: Experiment II

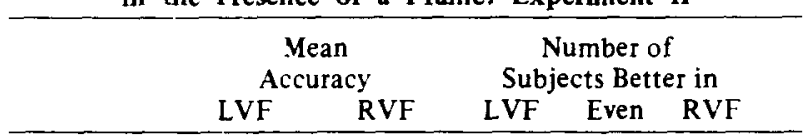

\begin{tabular}{llllll}
\hline Group 1* & & & & & \\
$\quad$ Men & 18.2 & 17.6 & 4 & 2 & 4 \\
Women & 18.1 & 15.0 & 6 & 0 & 4 \\
$\quad$ Total & 18.1 & 16.3 & 10 & 2 & 8 \\
Group 2** & & & & & \\
$\quad$ Men & 19.7 & 20.1 & 5 & 2 & 3 \\
Women & 18.1 & 18.0 & 5 & 1 & 4 \\
$\quad$ Total & 18.9 & 19.0 & 10 & 3 & 7 \\
\hline *Response card outside & & **Response card in T-scope
\end{tabular}

Examining the proportion of subjects showing a particular visual field superiority (Table 2 ), one again finds no signs of a laterality effect. Overall. 20 subjects were more accurate in the left visual field. and 15 more accurate in the right visual field.

This experiment indicates that the simple presence of a frame around the area in which the dots appear is not sufficient to produce a convincing laterality effect. The results of this experiment are in contradiction to those reported by Kimura (1969).

\section{EXPERIMENT III}

With a procedure such as that employed in the first two experiments. it is difficult to know whether any differences between visual fields are due to differences in the ability to localize dots or to differences in the ability to detect dots in one visual field rather than the other. The possibility of a difference in detection is suggested by the results of Filbey and Gazzaniga (1969). who found faster reaction times for the detection of dots in the left visual field. Kimura (1969) made an attempt to assess the role of detection processes. but used a very crude threshold procedure that may well have been insensitive to any differences. In addition. she tested detection and localization in different experiments, further weakening the adequacy of her test.

The present experiment involved simultaneous detection and localization procedures. employing a signal detection paradigm. The procedure was similar to that of the tirst experiment. except that a number of trials were introduced in which no dot appeared at all. The subject was asked to say "no" if no dot appeared. and to indicate where it was on a response card when a dor did appear. By considering attempted localizations as "yes" responses. it was possible to determine accuracy of detection. and by considering the localization responses. it was possible to determine accuracy of localization.

\footnotetext{
Method

Materials. Stimulus materials were those used in the first
} 
Table 3

Sample Matrix for Detection Calculations

\begin{tabular}{lll}
\hline & Yes Response & No Response \\
\hline Signal & Left response to & No response to \\
& Left dot $\left(\mathrm{p}_{1}\right)$ & Left dot $\left(\mathrm{p}_{2}\right)$ \\
\multirow{3}{*}{ No Signal } & Left response to & No response to \\
& No dot $\left(\mathrm{p}_{3}\right)$ & No dot $\left(\mathrm{p}_{4}\right)$ \\
\hline
\end{tabular}

experiment, except that the 12 buffer cards with dots falling outside the test area were omitted and a set of 24 blank cards was added to the 24 cards containing dots.

Subjects. The subjects were right-handed introductory psychology students at the University of Waterloo. Initially, 22 subjects were tested, but 2 subjects were dropped from the study because their false-alarm rate was greater than their hit rate. The final analyses were carried out on 20 subjects. 10 men and 10 women.

Procedure. The procedure followed the basic format of Group 2 in the first experiment. A single dot was exposed for $6 \mathrm{msec}-$ the shorter exposure duration being used to reduce detection accuracy-in one of the 24 positions indicated in Figure 1. After a $1-\mathrm{sec}$ delay. during which time the field was illuminated and the fixation point was visible, the response card appeared in the tachistoscope for $5 \mathrm{sec}$. Subjects were required to indicate whether or not a dot had been present. and if they thought it had been to indicate the number of the square corresponding to the position in which it had appeared. A response was required on every trial.

A total of 144 trials were given. in three blocks of 48 . Within each block, there were 24 trials on which the card was blank and 24 on which a dot appeared. The dot appeared once in each of the 24 possible locations in each block.

Scoring. A simple detection analysis does not permit one to derive separate detection scores for the two visual fields. since there is nothing in a yes-no response to indicate visual field, nor any way of assigning blank cards to the left or right visual field. For this reason. a procedure was adopted that required classifying each yes response as to visual field by also taking into account the location of the response square called by the subject.

If we do this, there are actually three different types of stimulus events: left-field dots, right-field dots, and blank cards. In addition, the subject may make three different responses: no, yes it was on the left. and yes it was on the right. This provides a total of nine possible stimulus-response contingencies. Now, suppose we consider the decision that a dot is in the left visual field and the decision that a dot is in the right visual field to be processes taking place along two different decision axes. Then, for left-field detection, a "hit" is a left visual field response to a dot presented in the left visual field, and a "false alarm" is the claim that a blank card shows a dot in the left visual field. A "miss" occurs when the subject claims that there is no dot when there actually is one in the left visual field. The fourth cell of the detection matrix is produced by the correct response "no" to a blank card. Another relevant possibility, of course, occurs when the subject gives a right-field response to a left-field dot. Here we must assume that the strength of the stimulus along the right-field decision axis exceeded that along the left decision axis. but we have no way of knowing whether or not the strength reached criterion on the left-field axis. In other words, it is quite possible for a stimulus to reach criterion along both axes. but for a right-field response to be given simply because it was the stronger of the two. For the present analysis, then, it was assumed that right-field responses could be ignored when determining a detection value for the left visual field, and vice versa. Thus. detection values for each field were calculated from matrices such as that shown in Table 3 .

It should be noted that, according to this interpretation, a right-field response to a left-field dot is merely a matter of the relative strength of signals along two decision axes. There seems to be no necessity for introducing a concept such as "visual allesthesia" (Pohl et al., 1972).
Because we know little about the underlying distributions of signal and noise and because the present experiment provides data based on relatively few observations. it was deemed best to use nonparametric estimates of sensitivity and bias. As a measure of sensitivity, an estimate of the area under the ROC curve. $p(\bar{A})$, was used (McNichol. 1972). As a measure of bias, the false-alarm rate ( $p_{3}$ in Table 3) was used (Richardson, 1972).

There are. in addition. two different logical measures of localization accuracy. One is simply a count of the number of dots correctly localized in each visual field. The other takes into account the likelihood of detecting dots presented in each visual field, and is the number of dots correctly localized in a given visual field divided by the number of dots presented in that visual field and detected. Both measures were used in analyzing the present data.

\section{Results}

In terms of correct localizations, the mean values follow the same pattern as that reported by Kimura (1969) (see Table 4). That is, men were somewhat better in the left visual field and women somewhat better in the right visual field. However, this effect does not even begin to approach statistical significance $[\mathrm{F}(1.18)=0.46]$. When these scores are corrected to take into account the accuracy of detecting the dots, any trends relating to localization completely disappear. In general, the men are more accurate than the women, although this effect is also not significant.

There were also no compelling differences between the two visual fields in the ease of detecting the dots (Table 5). However, there were very striking

Table 4

Accuracy of Localization in the Absence of a Frame: Experiment III

\begin{tabular}{|c|c|c|c|c|c|}
\hline & \multicolumn{2}{|c|}{$\begin{array}{c}\text { Mean } \\
\text { Accuracy }\end{array}$} & \multicolumn{3}{|c|}{$\begin{array}{c}\text { Number of } \\
\text { Subjects Better in }\end{array}$} \\
\hline & LVF & RVF & LVF & Even & RVF \\
\hline & \multicolumn{5}{|c|}{ Absolute Correct } \\
\hline Men & 9.90 & 9.00 & 6 & 1 & 3 \\
\hline Women & 6.80 & 7.90 & 5 & 0 & 5 \\
\hline \multirow[t]{2}{*}{ Total } & 8.35 & 8.45 & 11 & 1 & 8 \\
\hline & \multicolumn{2}{|c|}{ Percentage Localized } & Those & \multicolumn{2}{|c|}{ Detected } \\
\hline Men & 32.3 & 34.6 & 5 & 0 & 5 \\
\hline Women & 23.9 & 29.2 & 5 & 0 & 5 \\
\hline Total & 28.1 & 31.9 & 10 & 0 & 10 \\
\hline
\end{tabular}

Table 5

Dot Detectability in the Absence of a Frame: Experiment III

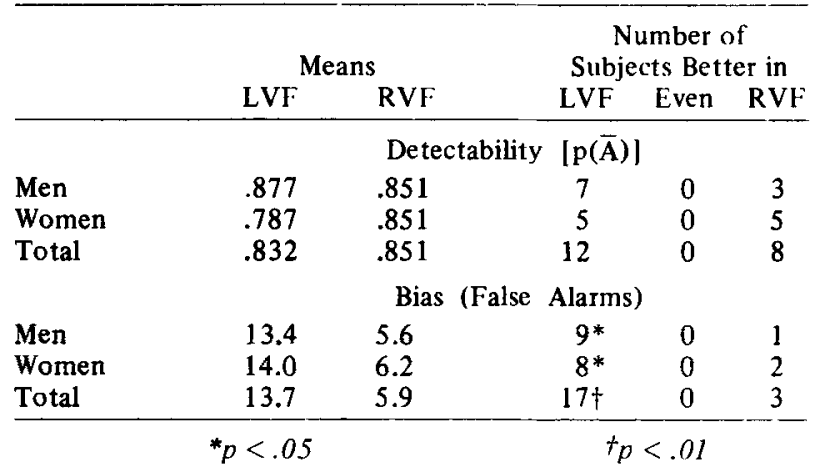


differences in response bias, as determined by the false-alarm rates. Nine of the 10 men and 8 of the 10 somen gave more left-tield responses than right-field responses in the absence of a dot $(p<.01)$.

This difference implies that subjects used a different criterion for the detection of dots in the left visual field than for the detection of dots in the right visual tield. In general, subjects were much more conservative about claiming a dot to be in the right visual field. If subjects show a lower criterion for guessing that a dot is in the left visual field, they will attempt to localize dots in the left visual field on the basis of minimal information, while waiting to be quite certain before attempting to localize a dot in the right visual field. Thus, dots which are not truly detected may be localized in the left visual field. If there are a limited number of response alternatives available, some of these false alarms will result in adventitiously correct localizations. Conversely, a rigorous criterion could reduce the number of right visual field localizations. In this way, a criterion shift could produce a left visual field superiority such as that reported by Kimura (1969) without indicating a superiority of localization within that field.

Basically, then, this experiment found no differences between visual field in either detection or localization, but did find a strong response bias to report dots as being in the left visual field. This may serve as a basis for artificially producing left visual field effects under some conditions.

\section{EXPERIMENT IV}

The previous experiment, involving signal detection procedures, showed a very striking response bias favoring giving left-field responses. Because of the different results that have been reported with and without frames of reference (Pohl et al., 1972), Experiment III was repeated using a frame of reference around the locations in which the dots could appear.

\section{Method}

Materials. Stimulus cards were the same as those used in the preceding experiment. except that the number of blank cards was reduced from 24 to 12 . The fixation card and the response card were those used in Experiment II. in which outline rectangles enclosed the areas in which dots could appear, and only 24 possible localizing responses were provided.

Subjects. The subjects were introductory psychology students at the University of Waterloo. paid for their participation in the experiment. All were right-handed. Twenty subjects, 10 men and 10 women, were tested.

Procedure. The procedure was virtually identical to that used in the preceding experiment. Dots were exposed for $6 \mathrm{msec}$, and following a 1-sec delay, in which both the fixation point and the trames were visible. the response card appeared in the tachisiuscope. As betore. the subjects were required to indicate whether or not a dot had been present, and if they thought it had been, to indicate the number of the square corresponding to the position in which it had appeared. A response was required on every tri..!
Table 6

Accuracy of Localization in the Presence of a Frame: Experiment IV

\begin{tabular}{|c|c|c|c|c|c|}
\hline & \multicolumn{2}{|c|}{$\begin{array}{c}\text { Mean } \\
\text { Accuracy }\end{array}$} & \multicolumn{3}{|c|}{$\begin{array}{c}\text { Number of } \\
\text { Subjects Better in }\end{array}$} \\
\hline & LVF & RVF & LVF & Even & RVF \\
\hline & \multicolumn{5}{|c|}{ Absolute Correct } \\
\hline Men & 24.1 & 21.5 & 6 & 0 & 4 \\
\hline Women & 20.1 & 20.4 & 4 & 2 & 4 \\
\hline \multirow[t]{2}{*}{ Total } & 22.1 & 20.9 & 10 & 2 & 8 \\
\hline & \multicolumn{5}{|c|}{ Percentage Localized of Those Detected } \\
\hline Men & 81.6 & 76.7 & 5 & 0 & 5 \\
\hline Women & 69.2 & 75.3 & 3 & 1 & 6 \\
\hline Total & 75.4 & 76.0 & 8 & 1 & 11 \\
\hline
\end{tabular}

A total of 108 trials was given to each subject, on 72 of which dots were presented. Trials were given in three blocks of 36 . In each block, one dot appeared in each of the 24 possible positions and 12 blank cards were presented.

Scoring. Scoring and data analysis were the same as that employed in Experiment III.

\section{Results}

As can be seen in Table 6 , there were again no significant effects with the measure of absolute correct localization. The men were slightly more accurate than the women, and were somewhat more likely to be better in the left visual field, but both effects were very small.

When the localization data are expressed in terms of the percentage of those localized that were actually detected, a trend towards a Sex by Visual Field interaction emerges $[F(1.18)=3.57, p<.10]$. In terms of mean performance, men were somewhat better in the right visual field. This is in agreement with Kimura's (1969) findings. However, this interaction is not strongly borne out by an examination of the performance of individual subjects, although a relatively high proportion of the women were more accurate in the right visual field.

Table 7 indicates that there were no significant differences in terms of either detection or bias, although the women did tend to show the same response bias favoring the left visual field that was observed in the preceding experiment.

Table 7

Dot Detectability in the Presence of a Frame: Experiment IV

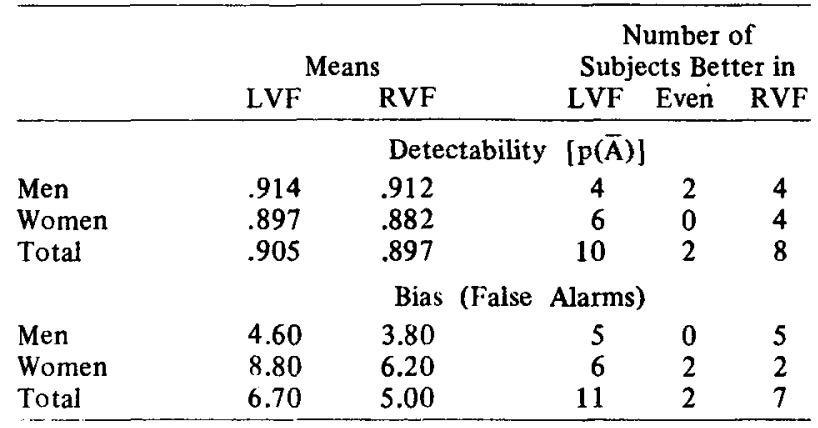




\section{DISCUSSION}

The present series of experiments have not clearly contirmed Kimura's (1969) finding of a left visual field superiority for dot localization. Nevertheless, of the 120 subjects tested in the four experiments, 64 were more accurate in the left visual field, while only 42 were more accurate in the right visual field $(p<.05)$. Left visual field superiorities, however, were consistently small, and certainly not sufficiently consistent to warrant the use of dot localization tests as a measure of right hemispheric function. Furthermore, the left visual field effect can be largely accounted for by a slight superiority of detection in the left visual field and by a bias for subjects to claim that dots appear in the left visual field. When localization accuracy is scored as a function of the number of dots correctly detected, the left field advantage disappears. Of the 40 subjects tested on the detection procedure, 18 showed more accurate localization in the left visual field and 21 in the right visual field. Thus, although Kimura's effect may be a real one, it is due not so much to hemispheric differences in spatial abilities as to differences in detection frequency and response bias.

No support was found for the Pohl et al. (1972) distinction between the presence or absence of a surrounding frame. Rather, the results of Bryden (1973), who reported no significant visual field effects, were basically substantiated.

Kimura (1969) also found sex differences in laterality. In her study, men showed a left visual field effect while women did not. The present study fails to replicate Kimura's sex difference. Laterality effects are similar for men and women. However, sex differences do emerge in two places. First of all. men are consistently more accurate in localization than are women. This male superiority, while never reaching statistical signiticance because of the small samples, is observed in every condition except the headmovement treatment of Experiment I. Second, in the detection experiments (Experiments III and IV), women show both poorer detectability and more frequent false alarms than do men. This finding would suggest a general tendency for women to be more liberal guessers than men.

The most clearly asymmetric effect found in this series of experiments was a tendency for subjects to give more false alarms in the left visual field than in the right. In the two detection experiments, 28 subjects gave more false alarms on the left, while only 10 gave more on the right $(\mathrm{p}<.01)$. While this effect was significant only in Experiment III, it persisted as a trend in Experiment IV. When a blank was presented, subjects were more likely to claim that a dot appeared on the left than that one appeared on the right. A similar effect was also seen in the first two experiments. in that right-field dots were more likely to be mislocalized in the left visual field than vice versa. In terms of signal detection theory. the increased false-alarm rate implies that subjects have a lower criterion for accepting that an item is present in the left visual field than that it is present in the right visual field. Perhaps one might say, then, that the right hemisphere is uncritical and will consider almost any signal as evidence that something is happening in the left visual field. This finding may relate to the frequently advanced idea that the left hemisphere is more analytic while the right hemisphere is more global and diffuse (Allard, 1972; Levy, 1973; Nebes. 1974).

One might also point out that the results of the present experiments indicate that the application of signal detection procedures to the study of hemispheric asymmetry may be fruitful. Frequently. different interpretations based on notions of differential sensitivity, attention. or bias are offered. and it is with just these distinctions that signal detection theory is concerned.

\section{REFERENCES}

Allard, F. Cerebral dominance. In M. Coltheart (Ed.). Readings in cognitive psychologv. Toronto: Holt. Rinehart. and Winston. 1972.

Blumstein. S. Goodglass. H., \& Tartter, J. The reliability of ear advantage in dichotic iistening. Brain and Language. 1975. 2. 226-236.

Bryden. M. P. Perceptual asymmetry in vision: Relation to handed ness. evedness. and speech lateralization. Cortex. 1973. 9. 418.4.35

Filbey. R. A.. \& Gazzaniga, M. S. Splitting the normal brain with reaction time. Psychonomic Science. 1969. 17. 335-336.

Heron. W. Perception as a function of retinal locus and attention. American Journul of Psychology' 1957. 70. 38-48.

Kimura. D. Spatial localization in left and right visual fields Canadian Journal of Psychology. 1969, 23, 445-458.

KinsBourne. $M$. The control of attention by interaction between the cerebral hemispheres. In $S$. Kornblum (Ed.). Attention and performance IV. New York: Academic Press. 1973.

LEVY, J. Lateral specialization of the human brain: Behavioral manifestations and possible evolutionary basis. In J. Kiger (Ed.). The biology of behavior. Corvallis. Oreg: Oregon State University Press. 1973.

MCNichol. D. A primer of signal detection theon: London: George Allen and Unwin. 1972.

Milner. B. Hemispheric specialization: Scope and limits In F. O. Schmitt \& F. G. Worden (Eds.). The neurosciences Third study program. Cambridge, Mass: M.I.T. Prcss. 1974.

NeBES, R. D. Hemispheric specialization in commissurotomized man. Psychological Bulletin. 1974. 81, 1-14.

Pohl. W.. Butters. N.. \& Goodglass. H. Spatial discrimination systems and cerebral lateralization. Cortex, 1972, 8. 305-314.

Richardson. J. T. E. Nonparametric indexes of sensitivity and response bias. Psychological Bulletin. 1972, 78, 429-432.

WhITE. M. Laterality differences in perception: A review. Psychological Bulletin. 1969. 72. 387-405.

White. M. Hemispheric asymmetries in tachistosonpic information-processing. British Journal of Psychology 19ᄀ2. 63. 497-508.

(Received for publication April 4. 1975: revision accepted August 15. 1975.) 